\title{
Early outcome of laparoscopic abdomino-perineal resection (APR) in low rectal and anal cancer
}

\begin{abstract}
Background: Colorectal cancer is the second most common malignancy in the western countries and the rectum is the most frequent site involved. Carcinoma in the lower part of the rectum involving anal canal especially sphincter or purely in the anal canal is now successfully managed by laparoscopic APR and postoperative morbidities are less and recovery is uneventful. In the current age of minimally invasive surgery, laparoscopic surgery for colon cancer has been established as equivalent to conventional open surgery in oncologic clearance. The advantages of laparoscopic surgery are smaller incisions, shorter recovery time and less wound related complications. However the narrow confines and angulations of the bony pelvis and the standard practice of autonomic nerve sparing total mesorectum excision has made laparoscopic surgery in the setting of rectal cancer more challenging.
\end{abstract}

Methods: This randomized controlled trial study was carried out in the Colorectal Surgery Unit, Department of Surgery, Bangabandhu Sheikh Mujib Medical University, Dhaka from May, 2012 to April, 2013. 25 patients were treated by conventional open APR and rest 25 patients were treated by laparoscopic APR. All Patients of two groups were selected by simple random method (lottery). Early outcome variables after surgery were evaluated.

Results: During early post-operative follow up, abdominal surgical site infection was found more in conventional open APR patients than that of laparoscopic APR patients $(p<0.05)$. Other morbidity and colostomy related complications were not significant in early post-operative period in both groups of population. Degree of pain was also less after laparoscopic APR. Only stoma function occurred earlier after Laparoscopic APR and ambulation, feeding liquid and feeding solid all had no difference for both group. Shorter mean length of postoperative total hospital stay and early hospital discharge was possible after Laparoscopic APR. Oncologic parameters were equivalent to those of open procedures.

Conclusion: The patients undergoing laparoscopic APR surgery had overall superior outcomes in term of surgical site infection, postoperative pain, postoperative total hospital stay and had equivalent oncologic clearance than those of open procedures in low rectal and anal cancer.

Keywords: early outcome, low rectal and anal canal cancer, laparoscopic abdominoperineal resection
Volume 2 Issue 5 - 2015

\author{
Rayhanur Rahman,' Shahadot Hossain \\ Sheikh, ${ }^{2}$ Rashidul Islam, ${ }^{2}$ Ismat Jahan Lima, ${ }^{2}$ \\ Ariful Alam, ${ }^{2}$ Gazi Muhammad Salahuddin, ${ }^{2}$ \\ Tariq Akhtar Khan ${ }^{2}$ \\ 'Pabna Medical College, Bangladesh \\ ${ }^{2}$ Department of Surgery, Bangabandhu Sheikh Mujib Medical \\ University, Bangladesh
}

Correspondence: Rayhanur Rahman, Assistant Professor of Surgery, Pabna Medical College, Pabna, Bangladesh, Tel 17| 1284791, Email tariqsurge@yahoo.com

Received: August 3, 2014 | Published: September 8, 2015

\section{Introduction}

Colorectal cancer is the second most common malignancy in the western countries and the rectum is the most frequent site involved. Carcinoma in the lower part of the rectum involving anal canal especially sphincter or purely in the anal canal is now successfully managed by laparoscopic APR and postoperative morbidities are less and recovery is uneventful. In the current age of minimally invasive surgery, laparoscopic surgery for colon cancer has been established as equivalent to conventional open surgery in oncologic clearance. The advantages of laparoscopic surgery are smaller incisions, shorter recovery time and less wound related complications. However the narrow confines and angulations of the bony pelvis and the standard practice of autonomic nerve sparing total mesorectum excision has made laparoscopic surgery in the setting of rectal cancer more challenging. ${ }^{1}$

\section{Aim and objectives}

This study was done to compare the early outcomes of laparoscopic APR surgery with that of open APR surgery in low rectal and anal cancer patients in term of incidence of surgical site infections, degree of postoperative pain by visual analogue scale, days of recovery of bowel function, postoperative total hospital stay time and margin clearance of tumor.

\section{Materials and methods}

This randomized controlled trial study was carried out in the Colorectal Surgery Unit, Department of Surgery, Bangabandhu Sheikh Mujib Medical University, Dhaka from May, 2012 to April, 2013. 25 patients were treated by conventional open APR and rest 25 patients were treated by laparoscopic APR. All Patients of two groups were selected by simple random method (lottery). Early outcome variables after surgery were evaluated.

\section{Results}

During early post-operative follow up, abdominal surgical site infection was found more in conventional open APR patients than that of laparoscopic APR patients $(\mathrm{p}<0.05)$. Other morbidity and colostomy related complications were not significant in early postoperative period in both groups of population. Degree of pain was also less after laparoscopic APR. Only stoma function occurred earlier after Laparoscopic APR and ambulation, feeding liquid and feeding solid all had no difference for both group. Shorter length of postoperative 
total hospital stay and early hospital discharge was possible after Laparoscopic APR. Oncologic parameters were equivalent to those of open procedures.

\section{Discussion}

Abdominal surgical site infection was absent in experimental group whereas it was present in $52 \%$ cases in control group in present study $(\mathrm{p}<0.05)$. But in case of perineal surgical site infection, there was no difference $(\mathrm{p}>0.05)$. But Fleshman et al. ${ }^{2}$ study showed that perineal infections occurred more often in the laparoscopic abdomino-perineal resection group ( 24 vs. 8 percent; $\mathrm{P}=0.02$ ). Only stoma function occurred earlier for experimental group $(\mathrm{p}<0.05)$ and ambulation, feeding liquid and feeding solid all had no difference for both group $(\mathrm{p}>0.05)$. Fleshman et al. ${ }^{2}$ had also observed dissisimilar recovery of bowel function among their study population. Comparison of colostomy related complications in both groups of patients were done. No patient developed early postoperative complications like hemorrhage and infection. Again no significant early colostomy related complications like prolapsed, retraction and necrosis were observed in both groups of population. Length of postoperative total hospital stay for experimental group was found shorter than that of control group. Early discharge was possible after laparoscopic APR (Table $1 \&$ Table 2). Laparoscopic APR was associated with a 50 per cent reduction in the length of hospitalization without any compromise to lateral or distal resection margins, number of lymph nodes harvested, or morbidity Iroatulama et al. ${ }^{3}$ Again, total hospital stay were significantly less in the study than in the control group Leung et al. ${ }^{4}$ Comparison of degree of postoperative pain between laparoscopic and open APR by visual analogue scale had done and analyzed. $88 \%$ of the patients had moderate pain- 2 in experimental group whereas $60 \%$ of the patients had worst pain in control group. ${ }^{5}$ Postoperative pain in experimental group was found to be less than that of control group $(\mathrm{p}<0.05)$. Postoperative analgesic requirements were significantly less in the study than in the control group Leung et al. ${ }^{3} \&$ Simon et al. ${ }^{1}$ Finally pathological evaluation in both groups of patients after histopathology following surgery had compared. Oncologic parameters were equivalent to those of open procedures in radial margin clearance $(\mathrm{p}>0.05)$ and in proximal margin clearance ( $>0.05) .{ }^{6}$ This study was similar to other study conducted by Fleshman et al. ${ }^{1}$

Table I Distribution of patients by demographic variables $(n=50)$

\begin{tabular}{|c|c|c|c|}
\hline \multirow{2}{*}{ Demographic variables } & \multicolumn{2}{|l|}{ Group } & \multirow{2}{*}{$P$ value } \\
\hline & Experimental & Control & \\
\hline \multicolumn{4}{|l|}{ Age in years } \\
\hline Mean (sd) & $38.72(0.89)$ & $42.60(15.70) \#$ & $0.397 * *$ \\
\hline Median (Min-Max) & $36(19-65)$ & $40(11-75)$ & \\
\hline \multicolumn{4}{|l|}{ Gender } \\
\hline Male & $15(60.0)$ & II (44.0)\# \# & $0.258 *$ \\
\hline Female & $10(40.0)$ & $14(56.0)$ & \\
\hline
\end{tabular}

*Chi-square test was done to measure the level of significance

** Mann-Whitney $U$ test was done to measure the level of significance

\# Figure within parentheses indicates minimum and maximum value in column

\# \# Figure within parentheses indicates percentage in column.

Table 2 Distribution of patients by outcome variables $(n=50)$

\begin{tabular}{|c|c|c|c|}
\hline \multirow{2}{*}{ Outcome variables } & \multicolumn{3}{|l|}{ Group } \\
\hline & Experimental & Control & $p$ value \\
\hline \multicolumn{4}{|l|}{ Morbidities } \\
\hline Abdominal surgical site infection & $0(0.0)$ & 13 (52.0) \#\# & $0.001 *$ \\
\hline Perineal surgical site infection & $5(20.0)$ & $6(24.0)$ & $0.733 *$ \\
\hline \multicolumn{4}{|l|}{ Colostomy related complications } \\
\hline Prolapse & $2(8.0)$ & I (4.0) \# \# & $1.000 *$ \\
\hline Retraction & I (4.0) & I (4.0) & $1.000 *$ \\
\hline Necrosis & $0(0.0)$ & I (4.0) & $1.000 *$ \\
\hline \multicolumn{4}{|l|}{ Pathological evaluation } \\
\hline Radial margin & $2(8.0)$ & $0(0.0) \# \#$ & $0.490 *$ \\
\hline Proximal margin & I (4.0) & $0(0.0)$ & $1.000 *$ \\
\hline \multicolumn{4}{|l|}{ Recovery of bowel function (Days) } \\
\hline Ambulation & $2(I-5)$ & $2(1-10) \#$ & $0.375^{* *}$ \\
\hline Stoma function & $2(2-5)$ & $2(2-10)$ & $0.017 * *$ \\
\hline Feeding liquid & $2(2-5)$ & $2(2-I I)$ & $0.070 * *$ \\
\hline \multirow[t]{2}{*}{ Feeding solid } & $2(3-6)$ & $2(3-12)$ & $0.070 * *$ \\
\hline & & & $0.001 *$ \\
\hline \multicolumn{4}{|l|}{ Degree of postoperative pain } \\
\hline Moderate pain-2 & $22(88.0)$ & I (4.0) \#\# & \\
\hline Severe pain & $3(12.0)$ & $9(36.0)$ & \\
\hline Worst pain & $0(0.0)$ & $15(60.0)$ & \\
\hline Postoperative hospital stay (Days) & $0.001 * *$ & & \\
\hline Mean (sd) & $10.96(3.45)$ & $16.28(5.08)$ & \\
\hline Median (Min-Max) & $10(7-19)$ & $15(9-27)$ & \\
\hline
\end{tabular}

$*$ Chi-square test is done to measure the level of significance.

** Mann-Whitney $U$ test is done to measure the level of significance. 


\section{Conclusion}

The patients undergoing laparoscopic APR surgery has overall superior outcomes in term of surgical site infection, postoperative pain, postoperative total hospital stay and has equivalent oncologic clearance than those of open procedures in low rectal and anal cancer.

\section{Acknowledgements}

None.

\section{Conflicts of interest}

The authors declare that there is no conflict of interest.

\section{References}

1. Simon SMN, Leung KL, Lee JFY. Laparoscopic assisted versus open abdominoperineal resection for low rectal cancer: a prospective randomized trial. Ann Surg Oncol. 2008;15(9):2418-2425.
2. Fleshman JW, Wexner SD, Anvari M, et al. Laparoscopic vs. open abdominoperineal resection for cancer. Dis Colon Rectum. 1999;42(7):930-939.

3. Iroatulama JN, Agachan $\mathrm{F}$, Alabaz $\mathrm{O}$, et al. Laparoscopic abdominoperineal resection for anorectal cancer. Am Surg. 1998;64(1):12-18

4. Leung KL, Kwok SP, Lau WY, et al. Laparoscopic assisted abdominoperineal resection for low rectal adenocarcinoma. Surg Endosc. 2000;14(1):67-70.

5. Row D, Weiser MR. An update on Laparoscopic resection for rectal cancer. Cancer Control. 2010;17(1):16-24.

6. Williams NS, Bulstrode CJK, O'Connell PR. Bailey and Love's Short Practice of Surgery. 25th edn. Edward Arnold London. 2008. p. 12301236. 